\title{
EXPLAINING THE INFRARED AND SUBMILLIMETER BACKGROUNDS BY DECAYING PARTICLES
}

\author{
Boqi Wang and George B. Field \\ Harvard-Smithsonian Center for Astrophysics \\ Cambridge, MA 02138 USA
}

\begin{abstract}
We propose that photons produced by decaying particles may account for the recently observed isotropic infrared background (IRB) between 1 and $5 \mu \mathrm{m}$. We find that for decaying particle masses $m_{x}$ between 5 and $18 \mathrm{keV}$, lifetime $\tau_{d}$ between 2 and $7 \times 10^{10} \mathrm{~s}$, and photon branching ratio $B_{\gamma}$ between 4 and $9 \times 10^{-4}$, the observed spectrum of decay photons is consistent with the observed spectrum of the IRB. The same parameters can explain the submillimeter background.
\end{abstract}

\section{INTRODUCTION}

In order to explain the isotropic radiation component at submillimeter wavelengths (Matsumoto et al. 1988), Fukugita (1988) proposed that particles with mass $m_{x}=1-10 \mathrm{keV}$ decay into photons with a small branching ratio $B_{\gamma}$. The model is constrained by direct observations of decay photons. In particular, Field and Walker (1989) showed that the proximity effect in the numbers of Ly $\alpha$ clouds (Bajtlik, Duncan, and Ostriker 1988) gives strong upper limits on the flux of photons with energies $>13.6 \mathrm{eV}$ in the rest frame. They proposed model parameters such that the decay photons would appear at wavelengths $\geq 1 \mu \mathrm{m}$. Matsumoto, Akiba, and Murakami (1988) recently observed diffuse celestial radiation at wavelengths between 1 and 5 $\mu \mathrm{m}$. Here we consider a scenario in which the peak of the decay photon spectrum is at several $\mu \mathrm{m}$. In the following we compare the observed spectrum of the IRB to that of decay photons, while constraining the calculations to ensure that the heat deposited in the electrons can explain the submillimeter background (SMB).

\section{DECAY PHOTON SPECTRUM AND THE OBSERVATIONS}

The SMB can be fitted by nonrelativistic Compton scattering with a Zeldovich-Sunyaev parameter $y_{c}=0.028 \pm 0.004$ (Hayakawa et al. 1987). This corresponds to an energy density $U_{S M B}=(4.8 \pm 0.9) \times 10^{-14} \mathrm{erg} \mathrm{cm}^{-3}$ at submillimeter wavelengths. Matsumoto, Akiba, and Murakami (1988) observed diffuse celestial light at various IR wavelengths between 1 and 5 $\mu \mathrm{m}$. The observed intensities are plotted in Figure 1. Here we propose that they are due to photons emitted by decaying massive particles, while the SMB is caused by inverse Compton scattering of the cosmic microwave background by electrons heated by the decay photons.

We calculate the spectrum of the decay photons, including the energy loss due to both expansion and Compton scattering. The free parameters are the mass of decaying particles $m_{x}$, the decay lifetime $\tau_{d}$, and the photon branching ratio $B_{\gamma}$. We fit the IR data with the calculated spectrum of decay photons, and impose the additional requirement $U_{a}-U_{I R B}=U_{S M B}$ (observed), where $U_{a}$ is the total available energy density from the decay photons. A typical example of a fit to the IRB is shown in Figure 1, where $m_{x}=10 \mathrm{keV}, \tau_{d}=3 \times 10^{10} \mathrm{~s}$ and $B_{\gamma}=9 \times 10^{-4}$. We find that there is a small region given by $m_{x}=3$ to $26 \mathrm{keV}$ and $\tau_{d}=1$ to $10 \times 10^{10} \mathrm{~s}$ which yields acceptable values for the spectrum of the IRB and for $U_{S M B}$. The corresponding values of $B_{\gamma}$ lie between 4 and $40 \times 10^{-4}$. 


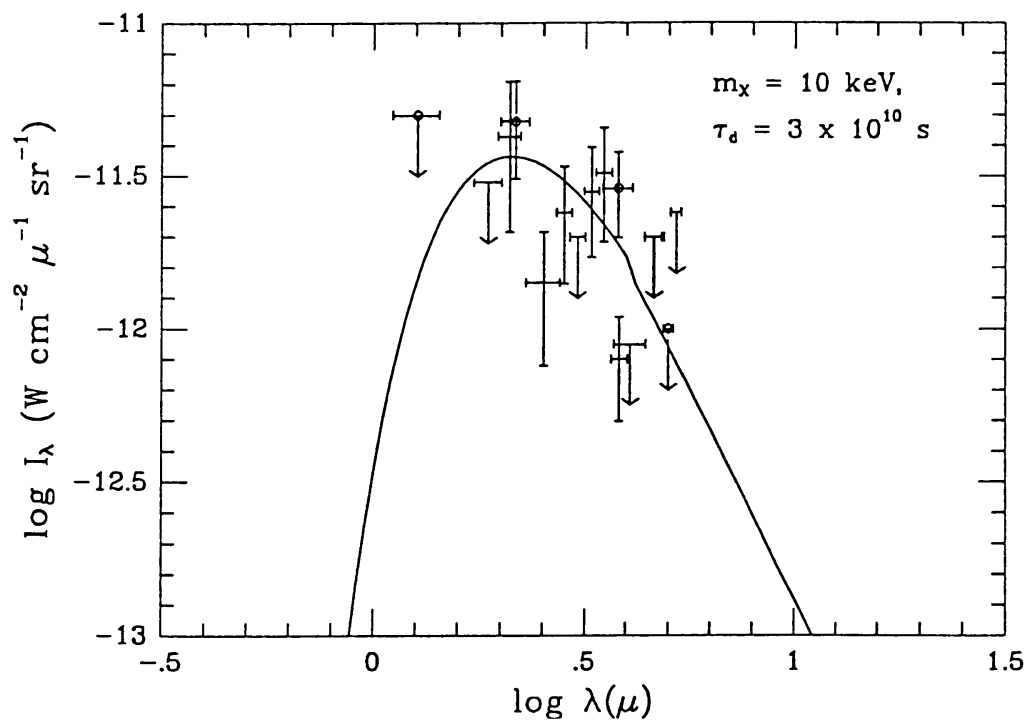

Figure 1. Calculated spectra $I_{\lambda}$ of decay photons compared with observations. The solid curve corresponds to the spectrum for $m_{x}=10 \mathrm{keV}, \tau_{d}=3 \times 10^{10} \mathrm{~s}$, and $B_{\gamma}=9 \times 10^{-4}$. The observed points are from Matsumoto, Akiba, and Murakami (1988).

\section{DISCUSSION}

Field and Walker (1989) showed that the proximity effect of Ly $\alpha$ absorption lines in quasar spectra (Bajtlik, Duncan, and Ostriker 1988) at redshift implies an ionization rate for hydrogen, $3.4 \times 10^{-12} \mathrm{~s}^{-1}$. The ionization rate caused by the decay photons discussed here reaches this value only for $z \geq 7$. Based on the lack of $\gamma$-rays from SN 1987A observed by the Gamma Ray Spectrometer on the Solar Maximum Mission, Kolb and Turner (1989) showed $\tau_{d} m_{x} / B_{\gamma} \geq 8 \times 10^{14} \mathrm{~s} \mathrm{keV}$ for $100 \mathrm{eV} \leqslant m_{x} \leq$ several MeV. This constraint substantially reduces the range of acceptable parameters to: $m_{x}=5-18 \mathrm{keV}, \tau_{d}=(2-7) \times 10^{10} \mathrm{~s}$, and $B_{\gamma}=$ (4-9) $\times 10^{-4}$. Constraints from stellar evolution models are less stringent than those from SN 1987A, unless $m_{x} \leqslant 60 \mathrm{eV}$ (Raffelt, Dearborn, and Silk 1988). The above ranges of parameters, consistent with other astrophysical constraints, implies that $z_{d}=(6-10) \times 10^{3}$, and that $\Omega_{x}$ $=0.07-0.14$.

Acknowledgment-This work is supported in part by the NASA grant NAGW931.

\section{REFERENCES}

Bajtlik, S., Duncan, R. C., and Ostriker, J.P. 1988, Ap. J., 327, 570.

Field, G. B. and Walker, T.P. 1989, submitted to Phys. Rev. Lett.

Fukugita, M. 1988, Phys. Rev. Lett., 61, 1046.

Hayakawa, S. et al. 1987, Publ. Astron. Soc. Japan, 39, 941.

Kolb, E. W. and Turner, M. S 1989, Phys. Rev. Lett., 62, 509.

Matsumoto, T. et al. 1988, Ap. J., 329, 567.

Matsumoto, T., Akiba, M., and Murakami, H. 1988, Ap. J., 332, 575.

Raffelt, B., Dearborn, D., and Silk, J. 1988, Ap. J., 336, 61. 
V.V. Burdyuzha: If decaying neutrinos are used to explain the excess radiation in the sub-mm range, must there then be big fluctuations in the background?

B. Wang: It's true that perturbations may grow before most of the decaying particles decay, thus producing fluctuations in the background. For the current model, since we only want to explain the spectral distortion of the cosmic microwave background and the infrared background spectrum, the isotropy problem is not important.

J. Peebles: Since your theory would predict that the universe is dominated by hot matter at modest redshifts, will you have a problem accounting for galaxy formation?

B. Wang: First of all, since $\Omega \sim 0.07$ to 0.14 , you still may have a matter dominated era up to redshifts of $1 / \Omega=7-15$. Galaxies may be formed during this early matter dominated era. We are currently investigating the implications of decaying particles in galaxy formation. As just mentioned, perturbations may grow during the period of particle decay. Later on, interaction between matter and photons emitted by decaying particles may further develop the perturbations.

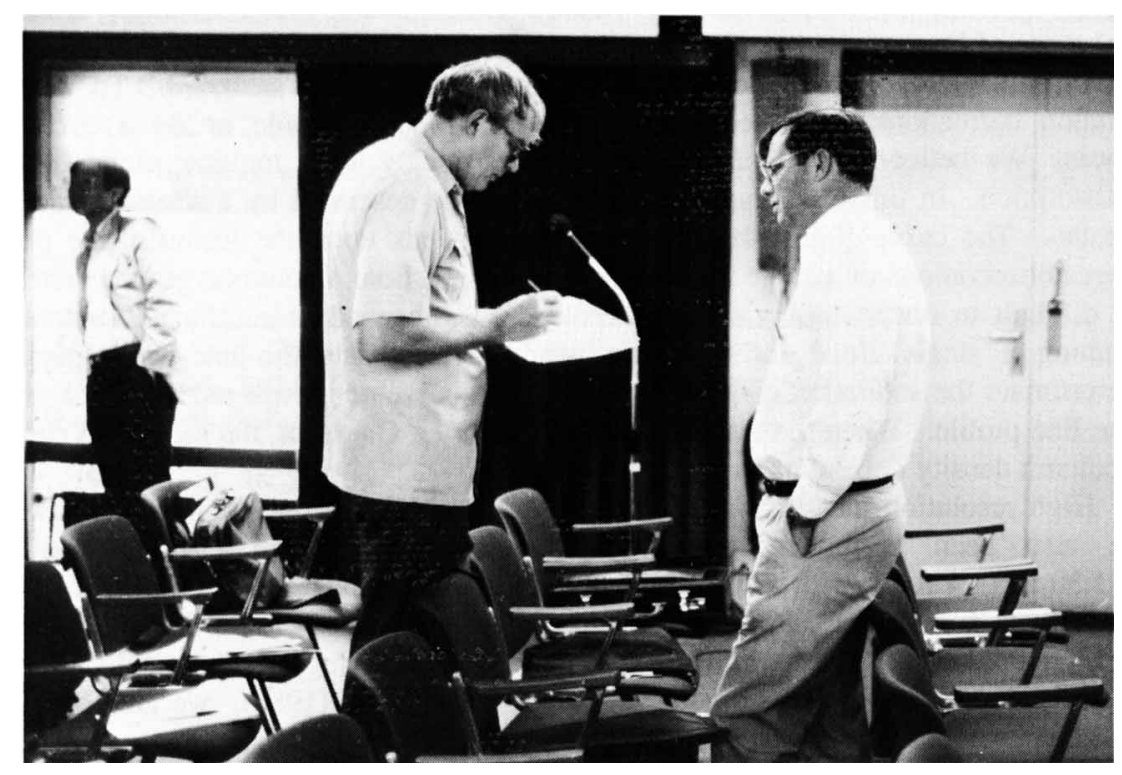

Stuart Bowyer and Bruce Partridge 